\title{
Use of Real-Time Hybrid Simulation to Evaluate the Performance-Based Seismic Design of Steel MRFs with Compressed...
}

Article · May 2010

DOI: $10.1061 / 41130(369) 207$

CITATION

1

4 authors, including:

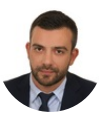

Theodore L. Karavasilis

University of Southampton

45 PUBLICATIONS 392 CITATIONS

SEE PROFILE
READS

76

Some of the authors of this publication are also working on these related projects:

Projec

Innovative seismic-resistant steel frame with high post-yield stiffness dampers for drift reduction View project

Multi-hazard resistant design and assessment of resilient post-tensioned self-centering steel frames View project 


\title{
Use of Real-Time Hybrid Simulation to Evaluate the Performance-Based Seismic Design of Steel MRFs with Compressed Elastomer Dampers
}

\author{
T.L. Karavasilis ${ }^{1}$, J.M. Ricles ${ }^{2}$, R. Sause ${ }^{2}$ and C. Chen ${ }^{3}$ \\ ${ }^{1}$ Department of Engineering Science, University of Oxford, Oxford OX1-3PJ, U.K. \\ ${ }^{2}$ ATLSS Engineering Research Center, Lehigh University, Bethlehem, PA 18015 \\ ${ }^{3}$ School of Engineering, San Francisco State University, San Francisco, CA 94132
}

\begin{abstract}
This paper presents an experimental program using real-time hybrid simulation to verify the performance-based seismic design of a two story, four-bay steel moment resisting frame (MRF) building with compressed elastomer dampers. The laboratory specimens, referred to as experimental substructures, are two individual compressed elastomer dampers, while the remaining part of the building is modeled as an analytical substructure. The proposed experimental technique enables an ensemble of ground motions to be applied to the building, resulting in various levels of damage, without the need to repair the experimental substructures since the damage is within the analytical substructure. Statistical experimental response results incorporating the ground motion variability show that an MRF with compressed elastomer dampers can be designed to perform better than a conventional steel special moment resisting frame (SMRF), even when the MRF with dampers is significantly lighter in weight than the conventional SMRF.
\end{abstract}

\section{INTRODUCTION}

Passive damping systems can significantly enhance the seismic performance of buildings by reducing inelastic deformation demands on the primary lateral load resisting system and by reducing drift, velocity, and acceleration demands on nonstructural components. Karavasilis et al. (2009a) evaluated the hysteretic behavior of an innovative compressed elastomer damper (Sweeney and Michael 2006) and based on the results of nonlinear dynamic history analyses found that steel moment resisting frames (MRFs) with compressed elastomer dampers can be designed to perform better than conventional special moment resisting frames (SMRFs), even when the MRF with dampers is significantly lighter in weight than the conventional SMRF.

To demonstrate and verify the full potential of new types of dampers, damper designs and performance-based design procedures for structural systems with dampers should be experimentally validated. Full-scale testing is a reliable but, at the same time, a challenging experimental technique. In particular, full-scale testing of structural systems designed to experience inelastic deformations may be cost and time prohibitive since the damaged components of the structural system need to be repaired or rebuilt after each test.

Real-time hybrid simulation combines physical testing and numerical simulation such that the dynamic performance of the entire structural system can be considered during the simulation. When real-time hybrid simulation is utilized to 
evaluate the performance of structures with rate-dependent damping devices, the damping devices may be tested as experimental substructures while the remaining part of the structural system is modeled analytically. The added benefit of this experimental technique is that it enables a large number of ground motions to be applied to the structure, resulting in various levels of damage, without the need to repair the test specimens since the damage will be within the analytical substructure.

This paper discusses an experimental program using real-time hybrid simulation to verify the performance-based seismic design of a two story, four-bay steel MRF building equipped with compressed elastomer dampers. The experimental substructures are two individual compressed elastomer dampers with the remaining part of the building modeled as an analytical substructure. The explicit unconditionally stable CR integration algorithm (Chen and Ricles 2008a, Chen et al. 2009a), a robust nonlinear finite element code (Karavasilis et al. 2009b) and an adaptive compensation scheme to minimize actuator delay (Chen and Ricles 2010) are integrated together and used in the real-time hybrid simulation to compute the structural response based on feedback restoring forces from the experimental and analytical substructures.

\section{STEEL MRFS WITH COMPRESSED ELASTOMER DAMPERS}

\section{Prototype building}

Figure 1(a) shows the plan view of the 2-story prototype office building used for the study. The study focuses on one typical perimeter MRF, which is comprised of four bays. This MRF is designed either as a conventional steel SMRF as defined in the 2006 International Building Code (ICC 2006), referred to herein as IBC 2006, or as a steel MRF equipped with compressed elastomer dampers. In the latter case, dampers and supporting diagonal braces are added to the two interior bays, as shown in Figure 1(b).

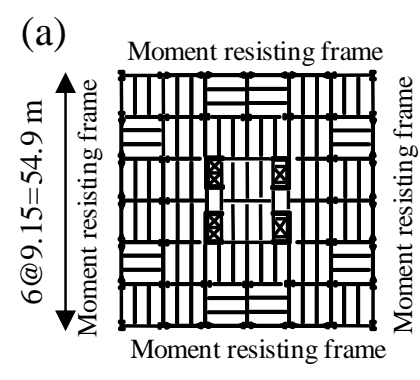

(b)

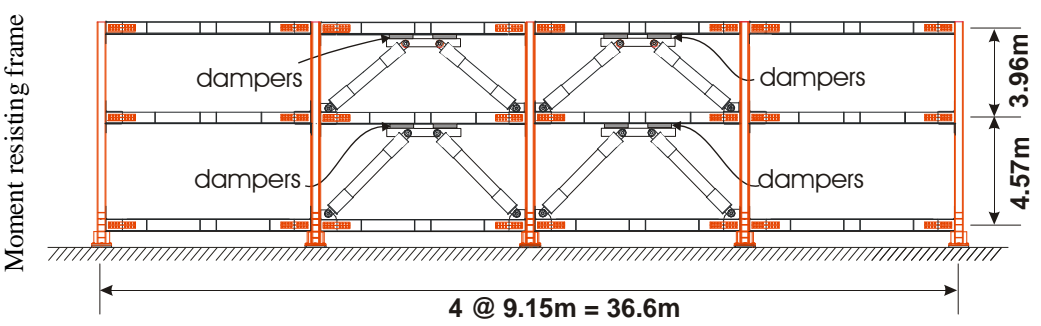

Figure 1. Prototype building structure: (a) plan view, and (b) perimeter MRF with dampers and diagonal bracing.

The members of the MRF are assumed to be A992 steel with a nominal yield stress of $345 \mathrm{MPa}$. The gravity loads considered in the design are those described in IBC 2006. A smooth design response spectrum with parameters $S_{\mathrm{DS}}=1.0, S_{\mathrm{D} 1}=0.6$, $T_{0}=0.12 \mathrm{sec}$ and $T_{\mathrm{s}}=0.6$ sec., defined by IBC 2006, represents the Design Basis Earthquake (DBE) which has a probability of exceedance of approximately $10 \%$ in 50 years. 


\section{Design of perimeter MRF as a conventional SMRF}

The perimeter MRF in Figure 1(b) is initially designed as a conventional SMRF using the equivalent lateral force procedure in the IBC 2006. This SMRF design without dampers, referred to herein as UD100V, satisfies the member strength criteria of the IBC 2006 with a response modification factor $R$ equal to 8 and also the $2 \%$ story drift limit of IBC 2006 with a deflection amplification factor $C_{\mathrm{d}}$ equal to 5.5 .

To study whether MRFs with compressed elastomer dampers can be designed to have less strength than a conventional SMRF (without dampers) but achieve similar or better levels of seismic performance, a perimeter MRF was designed without dampers using a design base shear equal to $0.50 \mathrm{~V}$, where $V$ is the design base shear for UD100V. The resulting MRF design, referred to herein as UD50V, does not satisfy the drift criteria of the IBC 2006. This MRF design is significantly lighter than UD100V.

Table 1 summarizes the properties of the two MRF designs, where the properties for UD50V are without the dampers. The table lists the column section, beam sections, steel weight, fundamental period of vibration, $T_{1}$, and the predicted maximum story drift, $\theta_{\max }$, under the DBE earthquake. The maximum story drift, $\theta_{\max }$, is determined on the basis of the equal displacement principle.

Table 1. Properties of MRF designs.

\begin{tabular}{c|c|c|c|c|c}
\hline MRF & $\begin{array}{c}\text { Column } \\
\text { Section }\end{array}$ & Beam Section & $\begin{array}{c}\text { Steel Weight } \\
(\mathrm{kN})\end{array}$ & $T_{1}(\mathrm{sec})$ & $\theta_{\max }(\%)$ \\
\hline \hline UD100V & W14x211 & $\begin{array}{c}1^{\text {st }} \text { story: W24x84 } \\
2^{\text {nd }} \text { story: W21x50 }\end{array}$ & 200 & 1.08 & 2.40 \\
UD50V & W14x120 & $\begin{array}{c}1^{\text {st }} \text { story: W24x55 } \\
2^{\text {nd }} \text { story: W18x40 }\end{array}$ & 124 & 1.48 & 3.23 \\
\hline
\end{tabular}

\section{Design of dampers for MRF}

The compressed elastomer structural dampers used in the study are fabricated by bonding four pieces of an elastomer (butyl rubber blend) onto a longitudinal steel bar, as shown in Figure 2(a). The pieces of elastomer on this bar are then pre-compressed into a steel tube (Figure 2(b)). Each prototype damper includes three tubes which are welded together (Figure 2(c)). To enable the damper to be attached to the structure, transverse bars with bolt holes are welded across the steel tubes and additional transverse attachment bars are welded across the narrow dimension of the longitudinal bars (Figure 2(c)).

The damper designs are based on the new generation of compressed elastomer dampers presented in Karavasilis et al. (2009a). The thickness and the area of these dampers are 4 times larger than the thickness and the area of the dampers used in the real-time hybrid simulations presented herein. The mechanical properties of these compressed elastomer dampers, namely the equivalent stiffness $\left(K_{\mathrm{eq}}\right)$ and loss factor $\left(\eta_{\mathrm{eq}}\right)$, were derived from the experimental data presented in Karavasilis et al. (2009a) and are given in Figure 3. Figure 3(a) shows that the stiffness $\left(K_{\mathrm{eq}}\right)$ decreases with increasing deformation, and slightly increases as the frequency increases for a given 
deformation. The sensitivity of $K_{\mathrm{eq}}$ to frequency diminishes as the deformation increases. Figure 3(b) shows that the loss factor $\left(\eta_{\mathrm{eq}}\right)$ is relatively constant for small amplitudes of deformation (less than $10 \mathrm{~mm}$ ) and significantly increases after slip of the elastomer occurs at about $15 \mathrm{~mm}$. There is a slight increase in $\eta_{\mathrm{eq}}$ as the frequency increases for given deformation.
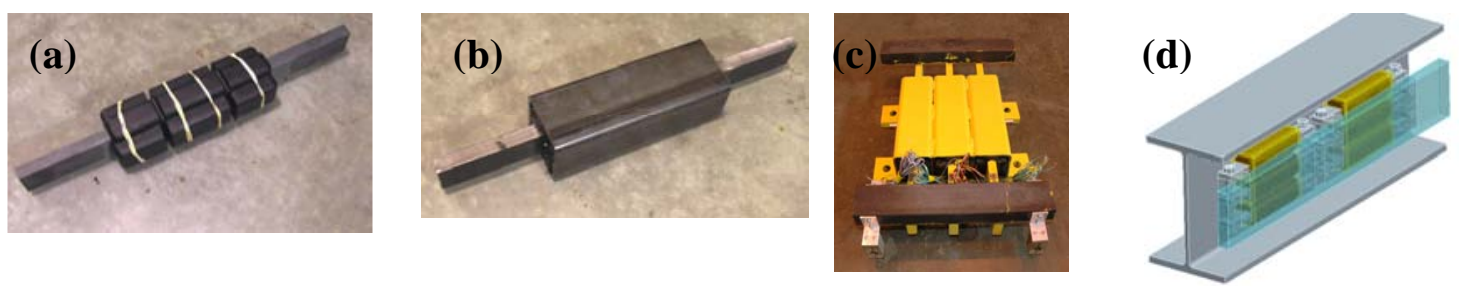

Figure 2. Fabrication of compressed elastomer damper: (a) elastomeric material wrapped around longitudinal bar; (b) elastomeric material and bar compressed into the steel tube; (c) damper bottom view with additional bolted transverse bars in place, and (d) installation to beam web
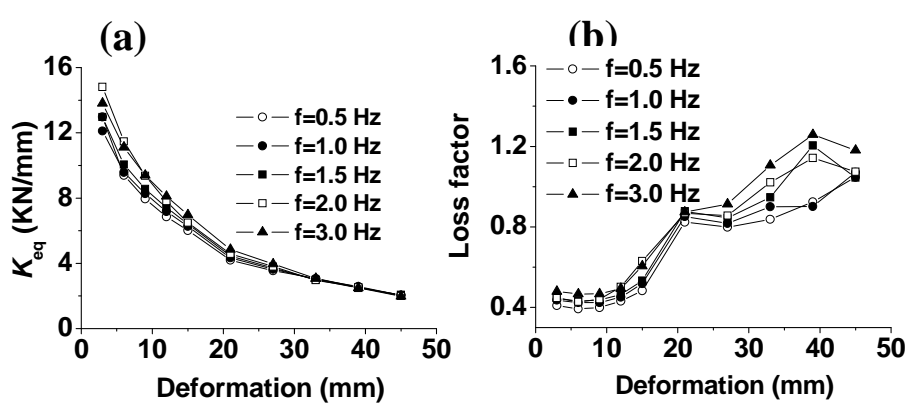

Figure 3. Mechanical properties evaluated from characterization tests: (a) equivalent stiffness, and (b) equivalent loss factor

The mechanical properties presented in Figure 3 are used to design the compressed elastomer dampers for the UD50V MRF with the aid of the simplified design procedure (SDP) developed by Lee et al. (2005). The SDP idealizes the damper hysteresis loops as linear viscoelastic ellipses and the damper design variables are the equivalent damper stiffness and the loss factor. More details on the SDP and the design of the compressed elastomer dampers for the UD50V MRF can be found in Karavasilis et al. (2009a). Under small deformations (less than $15 \mathrm{~mm}$ ) the damper hysteretic behavior resembles elastomeric behavior. When the deformation is larger than $15 \mathrm{~mm}$, slip of the elastomer compressed inside a steel tube occurs, and the hysteretic behavior is a combined elastomeric-frictional behavior.

Table 2 provides information for the UD50V MRF with dampers. The story drift and damper deformation demand estimates are for the DBE. It is observed that the UD50V MRF with 8 and 5 compressed elastomer dampers in the first and second stories, respectively, exhibits a significantly better anticipated performance ( $\theta_{\max }=$ $1.65 \%)$ than that of the conventional UD100V SMRF ( $\left.\theta_{\max }=2.40 \%\right)$. Moreover, the UD50V MRF with dampers has a steel weight equal to $124 \mathrm{kN}$ (UD50V) $+17.2 \mathrm{kN}$ 
(braces) $=141.2 \mathrm{kN}$, while the steel weight of the conventional UD100V SMRF is $200 \mathrm{kN}$.

Table 2. Design of UD50V MRF with compressed elastomer dampers.

\begin{tabular}{c|c|c|cc}
\hline $\begin{array}{c}\text { Brace steel Weight } \\
(\mathrm{kN})\end{array}$ & $\begin{array}{c}T_{1} \\
(\mathrm{sec})\end{array}$ & $\theta_{\max }(\%)$ & \multicolumn{2}{|c}{$\begin{array}{c}\text { No. Dampers per Story } \\
2^{\text {st }}\end{array}$} \\
\hline \hline 17.2 & 1.04 & 1.65 & 8 & 5 \\
\hline
\end{tabular}

\section{REAL TIME HYBRID SIMULATION}

\section{Real-time integrated control system architecture and analytical substructure modeling}

The performance of the MRF with compressed elastomer dampers is experimentally evaluated by conducting real-time hybrid simulations. The experimental substructures are two individual compressed elastomer dampers with the remaining part of the building modeled as an analytical substructure.

Since the dampers at a story level are placed in parallel in the prototype MRF (Figure 1(b)), they are subjected to the same velocity and displacement. Therefore, each of the dampers setups in the laboratory represents all of the dampers in one story. In a real-time hybrid simulation the measured restoring force from a compressed elastomer damper is multiplied by the number of dampers to obtain the total restoring force of all the dampers at a story level in the MRF.

As discussed previously, the thickness and the area of the elastomer of the dampers that are used in UD50V MRF are considered to be 4 times larger than the thickness and the area of the elastomer of the dampers in the experimental substructure. Consequently, in the real-time hybrid simulation the command displacement of the dampers was scaled down by a factor of 4 and the measured restoring force was amplified by a factor of 4 .

A nonlinear finite element code (Karavasilis et al. 2009b) has been implemented into the real-time integrated control system at the NEES Real-Time Multi-Directional (RTMD) Facility located at Lehigh University (Lehigh RTMD 2009). The architecture for the RTMD system is shown in Figure 4. A digital servo controller (RTMDctrl) with a $1024 \mathrm{~Hz}$ clock speed (sampling time $\delta \mathrm{t}=1 / 1024 \mathrm{sec}$ ) controls the motion of the servo-hydraulic actuators and is integrated with the realtime target workstation (RTMDxPC), simulation workstation (RTMDsim), and data acquisition workstation (RTMDdaq) using a shared common RAM network (SCRAMNet). SCRAMNet has a communication rate of about 180ns which enables the transfer of data among the integrated workstations in real-time with minimal communication delay. The nonlinear finite element code has been developed in a manner that enables the analytical substructure modeling, servo-hydraulic control law, and actuator compensation scheme to be integrated into a single SIMULINK model on the simulation workstation and then downloaded onto the target workstation using Mathworks xPC Target Software (MATLAB 2007). 
The model of the MRF has a total of 122 degrees of freedom and 71 elements. Inelastic behavior is modeled by means of a bilinear hysteretic lumped plasticity beam-column element with 3\% hardening and appropriate axial-moment yield surfaces. Diaphragm action is assumed at every floor level due to the presence of the floor slab. A lean-on column is used to model P- $\Delta$ effects on the MRF from gravity loads carried by gravity columns of the building.

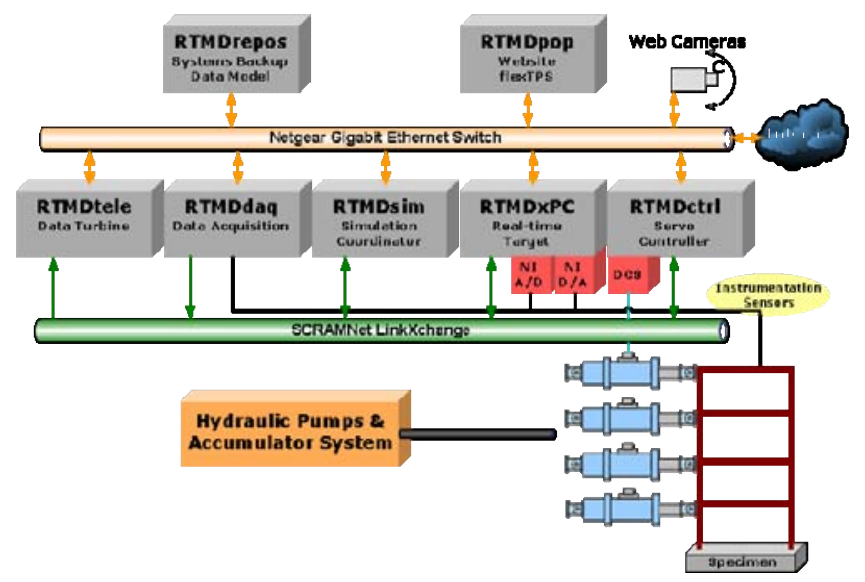

Figure 4. RTMD integrated control system architecture.

\section{Experimental substructure setup}

Figure 5 shows the experimental setup for the real-time hybrid simulation, which consists of the experimental substructures (two large-scale compressed elastomer dampers), two servo-hydraulic actuators with supports, roller bearings, and reaction frames. The two actuators have a load capacity of $2300 \mathrm{kN}$ and $1700 \mathrm{kN}$ with a maximum velocity of $840 \mathrm{~mm} / \mathrm{sec}$. and $1140 \mathrm{~mm} / \mathrm{sec}$, respectively, when three servo valves are mounted on each actuator. The servo-controller for the actuator used in the real-time hybrid simulations consists of a digital PID controller with a proportional gain of 20, integral time constant of 5.0 resulting in an integral gain of 4.0, differential gain of zero, and a roll-off frequency of $39.8 \mathrm{~Hz}$.

(a)

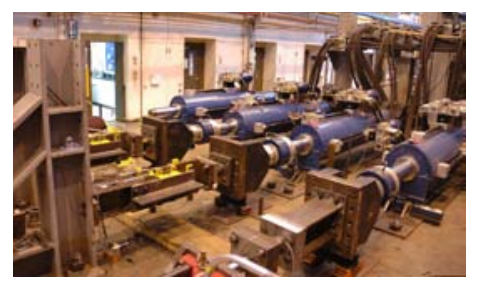

(b)

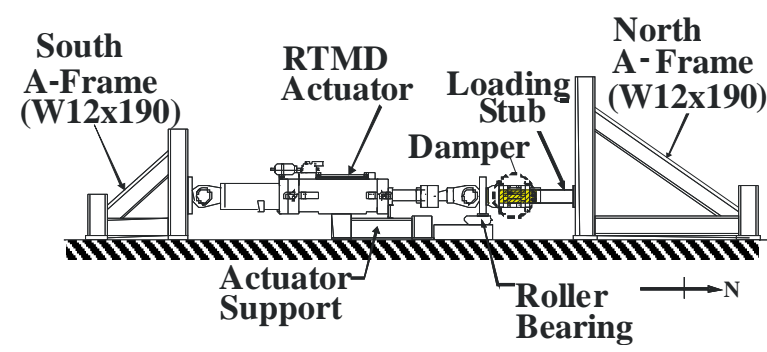

Figure 5. Compressed elastomer dampers: (a) photograph, and (b) details of test setup for each damper.

\section{Real-time integration of the equations of motion}

For the MRF structure with dampers shown in Figure 1(b), the temporal discretized equations of motion at the $i+1^{\text {th }}$ time step can be expressed as

$$
\boldsymbol{M} \cdot \ddot{\boldsymbol{x}}_{i+1}+\boldsymbol{C} \cdot \dot{\boldsymbol{x}}_{i+1}+\boldsymbol{r}_{i+1}^{a}+\boldsymbol{r}_{i+1}^{e}=\boldsymbol{F}_{i+1}
$$


where $\ddot{\boldsymbol{x}}_{i+1}$ and $\dot{\boldsymbol{x}}_{i+1}$ are the acceleration and velocity vectors of the structure, respectively; $\boldsymbol{r}_{i+1}^{a}$ and $\boldsymbol{r}_{i+1}^{e}$ are the restoring force vectors of the analytical and experimental substructures, respectively; $\boldsymbol{M}$ and $\boldsymbol{C}$ are the mass and damping matrices of the structure, respectively; and $\boldsymbol{F}_{i+1}$ is the excitation force.

The CR unconditionally stable explicit integration algorithm (Chen and Ricles 2008a, Chen et. al. 2009a) is used to solve Eq. (1) for the structural displacement vector $\boldsymbol{x}_{i+1}$. According to the CR algorithm, the variations of the displacement and velocity vectors of the structure over the integration time step $\Delta t$ are defined as

$$
\begin{gathered}
\dot{\boldsymbol{x}}_{i+1}=\dot{\boldsymbol{x}}_{i}+\Delta t \cdot \boldsymbol{\alpha}_{1} \cdot \ddot{\boldsymbol{x}}_{i} \\
\boldsymbol{x}_{i+1}=\boldsymbol{x}_{i}+\Delta t \cdot \dot{\boldsymbol{x}}_{i}+\Delta t^{2} \cdot \boldsymbol{\alpha}_{2} \cdot \ddot{\boldsymbol{x}}_{i}
\end{gathered}
$$

where $\boldsymbol{x}_{i}, \dot{\boldsymbol{x}}_{i}$ and $\ddot{\boldsymbol{x}}_{i}$ are the displacement, velocity and acceleration vectors of the structure at the $i^{\text {th }}$ time step, respectively; and $\alpha_{1}$ and $\alpha_{2}$ are matrices of integration parameters defined as

$$
\boldsymbol{\alpha}_{1}=\boldsymbol{\alpha}_{2}=4 \cdot\left(4 \cdot \boldsymbol{M}+2 \cdot \Delta t \cdot \boldsymbol{C}+\Delta t^{2} \cdot \boldsymbol{K}\right)^{-1} \cdot \boldsymbol{M}
$$

In Eq. (3) $\boldsymbol{K}$ is the initial stiffness matrix of the structure. It should be emphasized that this matrix includes the stiffness and damping contribution of the experimental substructures, i.e., the equivalent stiffness and damping of the two compressed elastomer dampers.

In real-time hybrid simulation, Eqs. (2.a) and (2.b) are used to obtain the velocity $\dot{\boldsymbol{x}}_{i+1}$ and displacement $\boldsymbol{x}_{i+1}$ vectors at the $i+1^{\text {th }}$ time step. The displacement vector $\boldsymbol{x}_{i+1}$ is decomposed into the analytical displacement vector $\boldsymbol{x}_{i+1}^{a}$ and the experimental (or command) displacement vector $\boldsymbol{x}_{i+1}^{e}$, which are imposed onto the analytical and experimental substructures, respectively, to obtain the restoring force vectors $\boldsymbol{r}_{i+1}^{a}$ and $\boldsymbol{r}_{i+1}^{e}$. Strictly speaking, $\boldsymbol{x}_{i+1}^{e}$ contains deformations, i.e., displacement differences of the nodes defining the connectivity of each of the experimental substructures. The analytical restoring force vector $\boldsymbol{r}_{i+1}^{a}$ is obtained with a standard nonlinear beam-column element state-determination procedure, while the experimental restoring force vector $\boldsymbol{r}_{i+1}^{e}$ is obtained from the feedback forces measured using load cells that are placed in each compressed elastomer damper test setup. The equilibrium Eq. (1) is then employed to calculate the acceleration response vector $\ddot{\boldsymbol{x}}_{i+1}$ at the $i+1^{\text {th }}$ time step, and the velocity $\dot{\boldsymbol{x}}_{i+2}$ and displacement $\boldsymbol{x}_{i+2}$ vectors for the next $i+2^{\text {th }}$ time step are readily available from Eqs. (2.a) and (2.b). This process is repeated to obtain the response over the whole duration of the earthquake ground motion.

Due to inherent servo-hydraulic dynamics, the actuator has an inevitable time delay in response to the displacement command. This time delay is usually referred to as actuator delay and will result in a desynchronization between the measured restoring forces from the experimental substructures and the integration algorithm in a real-time hybrid simulation. Studies on the effect of actuator delay (Wallace et al. 2005, Chen and Ricles 2008b) have shown that actuator delay is equivalent to creating negative damping and can destabilize a real-time hybrid simulation if not compensated properly. 
To minimize the detrimental effect of actuator delay during a real-time hybrid simulation, an adaptive inverse compensation (AIC) method was developed (Chen and Ricles 2010). The AIC method can be expressed using the following discrete transfer function that relates the compensated command displacement to the original command displacement

$$
G_{c}(z)=\frac{\left(\alpha_{e s}+\Delta \alpha\right) \cdot z-\left(\alpha_{e s}+\Delta \alpha-1\right)}{z}
$$

where in Eq. (4) $z$ is the complex variable in the discrete $z$-domain; $\alpha_{e s}$ is the estimated actuator delay constant; and $\Delta \alpha$ is an evolutionary variable with an initial value of zero. The AIC method uses an initial estimated $\alpha_{e s}$ for actuator delay compensation at the beginning of the hybrid simulation. The evolutionary variable $\Delta \alpha$ is used to adjust the initial estimate for $\alpha_{e s}$ to achieve accurate actuator control during a real-time hybrid simulation. The adaptation of the evolutionary variable $\Delta \alpha$ is based on a tracking indicator (Mercan 2007) which is defined as the enclosed area of the synchronized subspace plot between the actuator command displacement and the actuator measured response. Chen et al. (2009b) used the AIC method for real-time hybrid simulation of a structure with passive MR dampers, resulting in good actuator tracking.

\section{REAL-TIME HYBRID SIMULATION RESULTS}

An ensemble of 5 earthquake ground motions recorded on stiff soil sites (without near-fault effects) was used in the real-time hybrid simulations to evaluate the performance of the MRF with compressed elastomer dampers. The ground motions were scaled to the DBE and the Maximum Considered Earthquake (MCE) levels using the scaling procedure of Somerville (1997). The MCE has an intensity that is 1.5 times the DBE, and a $2 \%$ probability of exceedance in 50 years (FEMA 2000). Table 3 provides the scale factors and information for the 5 ground motions.

Table 3. Ground motions used in real-time hybrid simulations.

\begin{tabular}{l|l|c|c}
\hline \multirow{2}{*}{ Earthquake } & \multicolumn{2}{|c|}{ Station/Component } & \multicolumn{2}{c}{ Scale Factor } \\
\cline { 3 - 4 } & & DBE & MCE \\
\hline \hline Loma Prieta 1989 & Hollister/HSP090 & 1.99 & 2.99 \\
Manjil 1990 & Abbar/Abbar-T & 0.96 & 1.44 \\
Northridge 1994 & N Hollywood/CWC270 & 1.70 & 2.56 \\
Chi Chi 1999 & TCU049/TCU049-E & 1.92 & 3.67 \\
Chi Chi 1999 & TCU105/TCU105-E & 2.45 & 2.89 \\
\hline
\end{tabular}

Time history results from the real-time hybrid simulations are presented for the HSP090 record. The hysteresis of the compressed elastomer dampers is presented in Figure 6. The dampers are able to undergo numerous seismic induced deformation cycles without degradation of their behavior. Under the DBE the dampers at both floors exhibit an elastomeric behavior with fairly rounded peaks. Under the MCE the damper at the second floor develops some minor slip, while the damper at the first 
floor experiences an elastomeric-frictional behavior with slip that results in permanent deformation, but the damper maintains its energy dissipation capacity.

(a) Second floor damper (DBE)

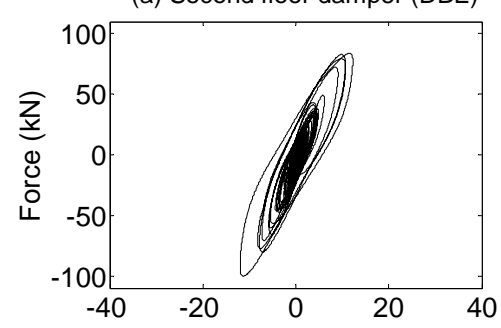

(b) First floor damper (DBE)

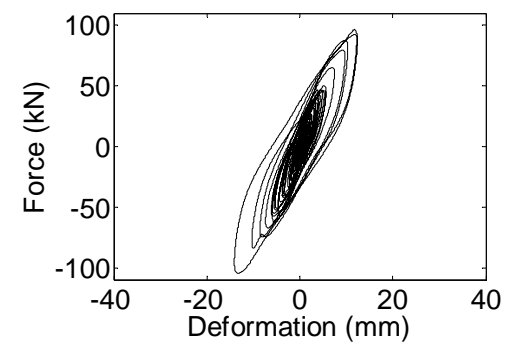

(c) Second floor damper (MCE)

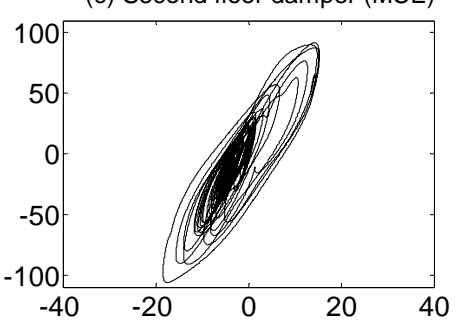

(d) First floor damper (MCE)

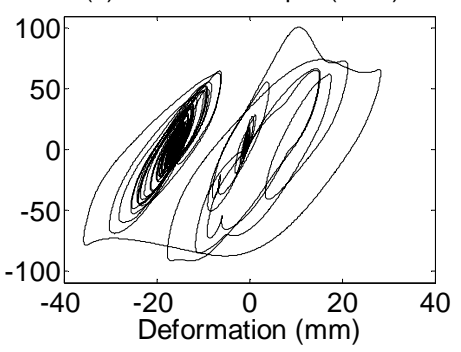

Figure 6. Damper hysteresis from real-time hybrid simulation.

Figure 7 shows the floor displacement time history of the MRF with dampers, UD50V MRF. Also presented in Figure 7 is the floor displacement time history of the conventional UD100V SMRF from a numerical analysis. The real-time hybrid simulation results show that the lighter UD50V MRF with dampers experiences significantly lower transient and residual story drifts than the conventional UD100V SMRF. Under the DBE the UD50V MRF with dampers has negligible story drift since the dampers do no slip and the frame remains essentially elastic. Under the MCE the dampers act as sacrificial elements, which develop permanent deformation due to slip, however, the dampers can be replaced after the earthquake. Some modest yielding occurs in the beams and at the ground level of the columns.

Table 4 presents the median experimental response values for the maximum story drift, $\theta_{\mathrm{max}}$; beam maximum plastic hinge rotation $\theta_{\mathrm{pl}, \mathrm{bm} \_ \text {max }}$; column maximum plastic rotation $\theta_{\text {pl,cl_max }}$; maximum floor absolute velocity $v_{\max }$; and floor absolute acceleration $a_{\max }$ of UD50V MRF from the real-time hybrid simulations. Also presented in Table 4 are the median values of the same response quantities for the conventional UD100V SMRF from numerical analysis. Table 4 shows that for the DBE the median $\theta_{\max }$ value of $1.40 \%$ for the MRF with dampers is slightly less than the $\theta_{\max }$ design demand of $1.65 \%$ used in the SDP (Table 2), while the $\theta_{\max }$ value of $2.60 \%$ for the UD100V SMRF is slightly larger than the $\theta_{\max }$ design demand of $2.40 \%$ according to the equal displacement principle (Table 1). It is also observed that the MRF with dampers has a significantly better performance than the UD100V SMRF in terms of the maximum story drift, plastic hinge rotations, absolute floor velocities and accelerations. Decreases in plastic hinge rotations in UD50V compared to UD100V are approximately 75\% and 57\% for the DBE and MCE, respectively. 
(a) Second floor (DBE)

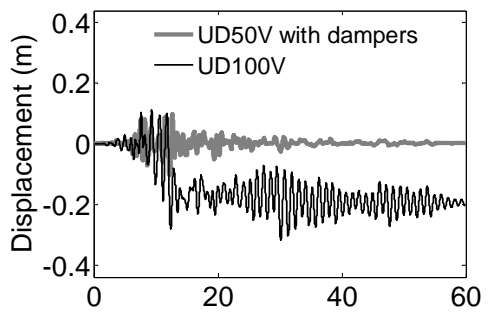

(b) First floor (DBE)

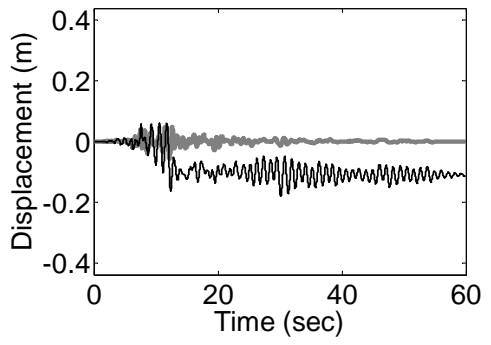

(c) Second floor (MCE)

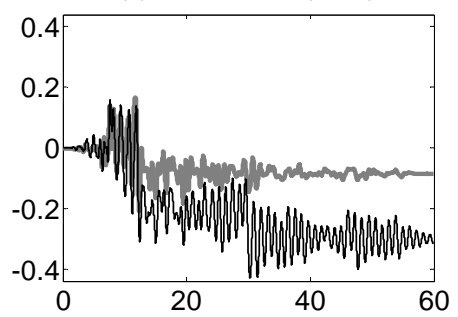

(d) First floor (MCE)

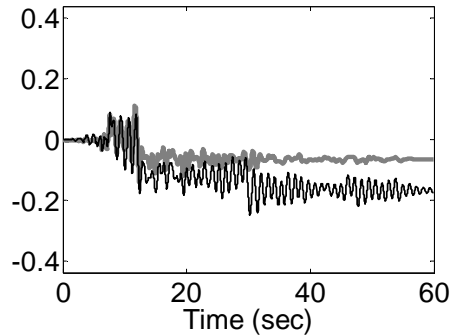

Figure 7. Floor displacement time history from real-time hybrid simulation, UD50V MRF subject to Loma Prieta earthquake record.

Table 4. Median values of response parameters.

\begin{tabular}{c|c|c|c|c|c|c|c|c|c|c|c}
\hline \multirow{2}{*}{ Design } & \multicolumn{2}{|c|}{$\theta_{\max }(\%)$} & \multicolumn{2}{c|}{$\theta_{\mathrm{pl}, \mathrm{bm} \max }(\%)$} & \multicolumn{2}{c|}{$\theta_{\mathrm{pl}, \mathrm{cl} \max }(\%)$} & \multicolumn{2}{c|}{$v_{\max }(\mathrm{m} / \mathrm{s})$} & \multicolumn{2}{c}{$a_{\max }\left(\mathrm{m} / \mathrm{s}^{2}\right)$} \\
\cline { 2 - 12 } \multicolumn{2}{c|}{} & DBE & MCE & DBE & MCE & DBE & MCE & DBE & MCE & DBE & MCE \\
\hline \hline \multirow{2}{*}{ UD50V } & Story 1 & 1.35 & 2.50 & 0.002 & 0.006 & 0.002 & 0.010 & 0.61 & 0.90 & 4.18 & 5.70 \\
& Story 2 & 1.40 & 1.80 & 0.000 & 0.000 & 0.000 & 0.000 & 0.77 & 1.10 & 5.16 & 6.50 \\
\hline \multirow{2}{*}{ UD100V } & Story 1 & 2.60 & 2.90 & 0.008 & 0.014 & 0.010 & 0.015 & 0.78 & 1.00 & 5.32 & 6.60 \\
\cline { 2 - 12 } & Story 2 & 2.40 & 2.60 & 0.000 & 0.003 & 0.000 & 0.000 & 1.11 & 1.28 & 5.66 & 6.36 \\
\hline
\end{tabular}

\section{SUMMARY AND CONCLUSIONS}

An experimental program using real-time hybrid simulation to verify the performance-based seismic design of a steel MRF equipped with compressed elastomer dampers was presented. The experimental substructures consist of two individual large-scale compressed elastomer dampers with the remaining part of the building modeled as an analytical substructure. The real-time hybrid simulations allowed an ensemble of ground motions to be applied to the structure resulting in various levels of damage, without the need to repair the test specimens since the damage was within the analytical substructure. Statistical experimental response results show that a steel MRF with compressed elastomer dampers can be designed to perform better than a conventional steel SMRF, even when the MRF with dampers is significantly lighter in weight than the conventional SMRF.

\section{ACKNOWLEDGEMENTS}

This paper is based upon work supported by grants from the Pennsylvania Department of Community and Economic Development through the Pennsylvania Infrastructure Technology Alliance, and by the National Science Foundation under 
Grant No. CMS-0402490 within the George E. Brown, Jr. Network for Earthquake Engineering Simulation Consortium Operation. The compressed elastomer dampers were manufactured and donated to the research project by Corry Rubber.

\section{REFERENCES}

Chen, C., Ricles, J.M. (2008a). "Development of direct integration algorithms for structural dynamics using discrete control theory." J. of Eng. Mechanics, ASCE, 134(8): 676-683.

Chen, C. and Ricles, J.M. (2008b). "Stability analysis of SDOF real-time hybrid testing systems with explicit integration algorithms and actuator delay using." Earthquake Eng. and Structural Dyn. 37(4):597-613.

Chen, C., Ricles, J.M., Marullo, T.M., Mercan, O. (2009a). "Real-time hybrid testing using the unconditionally stable explicit CR integration algorithm.” Earthquake Eng. and Structural Dyn., 38(1):23-44.

Chen, C., Ricles, J.M., Karavasilis, T., and Sause, R. (2009b). "Real-time hybrid simulation system for investigation of structural response under earthquake loading.” J. of Structural Eng., ASCE, in review.

Chen, C. and Ricles, J.M. (2010). "Tracking error-based servo-hydraulic actuator adaptive compensation for real-time hybrid simulation.” J. of Structural Eng., ASCE, 136(4), 2010.

FEMA. (2000), NEHRP Recommended Provisions for Seismic Regulations for New Buildings and Other Structures. Part 1 - Provisions and Part 2 - Commentary, FEMA 450, Federal Emergency Management Agency, Washington, D.C.

International Code Council (ICC). (2006). International Building Code, Falls Church, VA.

Karavasilis, T.L., Sause, R., Ricles, J.M. (2009a). "Seismic design and evaluation of steel MRFs with compressed elastomer dampers." Earthquake Eng. and Structural Dyn., in review.

Karavasilis, T.L., Ricles, J.M., Marullo, T., Chen, C. (2009b). “HybridFEM. A program for nonlinear dynamic time history analysis and real-time hybrid simulation of structures.” ATLSS Eng. Research Center Report 09-08.

Lee, K.S., Fan, C.P., Sause, R., Ricles, J. (2005). "Simplified design procedure for frame buildings with viscoelastic or elastomeric structural dampers.” Earthquake Eng. and Structural Dyn., 34:1271-1284.

Lehigh RTMD Users Guide. (2009). http://www.nees.lehigh.edu/index.php?page=rtmd-user-s-manual.

MATLAB. (2007). The Math Works, Inc., Natick, MA.

Mercan, O. (2007). "Analytical and experimental studies on large scale, real-time pseudodynamic testing.” PhD. Dissertation, Dept. of Civil and Environmental Eng., Lehigh Univ., Bethlehem, PA.

Somerville P. (1997). "Development of Ground Motion Time Histories for Phase 2 of the FEMA/SAC Steel Project.” Report No. SAC/DB-97/04, Sacramento, CA.

Sweeney, S.K., Michael, R. (2006). "Collaborative product realization of an innovative structural damper and application.” Proc. of IMECE2006, ASME Inter. Eng. Congress and Exposition, Chicago, Illinois, U.S.A. 
Wallace, M.I., Sieber, J., Neild, S.A., Wagg, D.J., Krauskopf, B. (2005). “Stability analysis of real-time dynamic substructuring using delay differential equation models.” Earthquake Eng. and Structural Dyn., 34(15):1817-1832. 